\title{
Macroscopic Modeling and Simulation of Freeway Traffic Flow
}

\author{
Jan Hueper, Gunes Dervisoglu, Ajith Muralidharan, Gabriel Gomes, Roberto Horowitz and Pravin Varaiya
}

\begin{abstract}
This paper illustrates the macroscopic modeling and simulation of Interstate 80 Eastbound Freeway in the Bay Area. Traffic flow and occupancy data from loop detectors are used for calibrating the model and specifying the inputs to the simulation. The freeway is calibrated based on the Link-Node Cell Transmission Model and missing ramp flow data are estimated using an iterative learning-based imputation scheme. An adhoc, graphical comparison-based fault detection scheme is used to identify faulty measurements. The simulation results using the calibrated model exhibit good agreement with loop detector measurements with total density error of $3.3 \%$ and total flow error of $7.1 \%$ over the 23 mile stretch of the freeway under investigation and the particular day for which the ramp flows were imputed.
\end{abstract}

\section{INTRODUCTION}

Today's situation of congested road-networks is a severe problem which has to be addressed due to the increasing trend of transportation demand every year. Operations planning, which includes ramp metering, demand and incident management, and its benefit assessment depend on the tools which successfully simulate the traffic flows in agreement with empirical data. This paper illustrates the macroscopic modeling, calibration and simulation of traffic flow on Interstate 80 Eastbound on a stretch of 23 miles in Northern California, extending from the Bay Bridge up to the Carquinez Bridge.

Traffic modeling is a field of research and public interest, since the number of motor vehicles is found to exceed the service capacity of provided roadway facilities, especially during periods of high demand such as morning and evening commute. There are two methods in traffic modeling, which essentially differ in the degree of resolution, i.e. in the level of detail of the modeled objects and their degrees of freedom. Microscopic traffic models model the dynamics of individual vehicles using the interactions between the vehicles and their vicinity, whereas macroscopic models use less detailed models and represent the traffic as a compressible fluid with the main properties flow, density and speed. The first order Macroscopic Cell Transmission Model (CTM) was adopted in this study [1],[2].

J. Hueper is with the Institute for Transport- und Automatisierungstechnik, Leibniz Universitaet Hannover. jan.hueper@googlemail.com

G. Dervisoglu is with the Department of Mechanical Engineering, University of California, Berkeley. gunesder@berkeley. edu

A. Muralidharan is with the Department of Mechanical Engineering, University of California, Berkeley. a jith@berkeley.edu

G. Gomes is a Researcher at California PATH. gomes apath. berkeley.edu

R. Horowitz is a Professor at Department of Mechanical Engineering, University of California, Berkeley. horowitz@berkeley.edu

P. Varaiya is a Professor at Department of Electrical Engineering, University of California, Berkeley. varaiya@eecs.berkeley.edu
The main advantage of Macroscopic traffic models over microscopic models is the significantly lower computational costs due to lower complexity. The decision to rely on macroscopic freeway modeling derives from the fact that this approach, which is closely related to the wave theory, is comparably easy to implement in software tools. Usually, the software is fast to run, which is a desirable feature, considering the fact that it is intended to allow users to run a large number of different scenarios in a short time [3]. Although complexity is low, the essentials of traffic behavior can accurately be reflected.

The density and flow data required for model specification is readily available for California freeways via loop detector based vehicle detector stations (VDS). The PeMS database [4] archives the flow, occupancy and speed data from these VDS. However, a common problem encountered is the quality (correctness) of mainline flow and density data used for modeling and imputation of missing ramp flows. Hence some corrections are necessary to ascertain healthy calibration. A basic fault detection scheme based on graphical comparison is elaborated in the subsequent sections.

This paper demonstrates the modeling and calibration procedure and presents the simulation results which show significant resemblance to observed congestion patterns on the modeled freeway section of Interstate 80 Eastbound in the Bay Area.

\section{THE MACROSCOPIC MODEL}

The model used for simulation is a modified version of Daganzo's Cell Transmission Model [1], named the LinkNode Cell Transmission Model (LN-CTM)[3]. It represents the freeway as a directed graph of links and nodes (Figure 1), where every link represents a road segment and the nodes represent junctions between the links. The flow exchange takes place at the nodes only and is indicated by a time varying split-ratio matrix, which specifies the portion of traffic moving from a particular input link to an output link. The nodes specify the locations where the freeway merges with an on-ramp or off-ramp and each node contains a maximum of one of each ramp. The links that introduce traffic flow into the network are called source links and links that accept flow out of the network are called sinks. In this respect, the off-ramps are sinks and the on-ramps are source links. It is assumed that off-ramps are always in free flow, i.e. they can accept flow from the mainline without any restriction.

The capacity $\mathrm{F}$, the free flow speed $\mathrm{v}$, the congestion wave speed $\mathrm{w}$, the critical density $n^{c}$ and the jam density $n^{J}$ of 


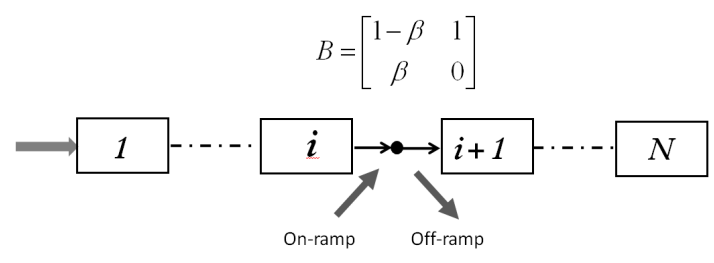

Fig. 1. Graphical representation of the freeway

each link are specified by its fundamental diagram (Figure 2) which are calibrated for each link based on PeMS data.

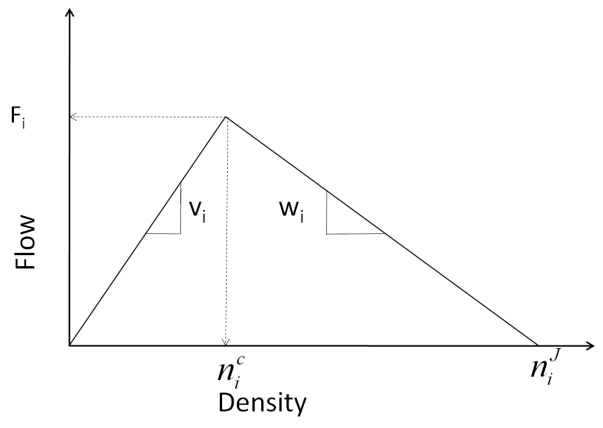

Fig. 2. Fundamental diagram for a freeway section.

The LN CTM can be simplified into a four mode switching model for analysis of freeway traffic flow [5]. The density updates and flow calculations can be expressed in closed form for these modes. The modes are distinguished by the flow condition before and after a node. Those conditions can be free-flow (F) or congestion (C). Hence, the four modes are called FF, CF, CC and FC, where the first and second letters correspond to the entering and exiting flows respectively. The total input demand for link $i, c_{i-1}(k)$, comprises all vehicles from the previous link moving with free flow speed, minus the vehicles that leave the freeway over the off-ramp, indicated by the split ratio; plus the vehicles that intend to enter the freeway over the on-ramp. The question whether the flow condition in the input node to link $i$ (or the output node from link $i-1$ ) is congested can be answered by comparing the calculated demand $c_{i-1}(k)$ with the downstream capacity, which is the maximum flow that can enter link $i$. If $c_{i-1}(k)$ exceeds the output capacity, link $i$ is in congestion. Once the modes for the links are determined, the densities can be calculated using the given demands, the densities from the previous period and the parameters from the fundamental diagram of each link.

\section{TRAFFIC MEASUREMENT}

The PeMS database archives the measurements from inductive loops installed on California freeways. The only direct measurements are the number of cars which cross a detector station and the fraction of time a vehicle is present over the loop. PeMS uses these values and calculates several other important measures like:
Occupancy: Percentage of time, when the detector is occupied, i.e. a car is above it.

Flow: Number of cars which pass a detector in a given time period.

Speed: Calculated using a G-factor and the flow and occupancy values. The G-factor is a combined factor of the average length of the vehicles traveling over the detector and the tuning of the detector.

Vehicle Hours Traveled (VHT): Amount of time that all the vehicles spent on a certain section of freeway over a certain period of time.

Vehicle Miles Traveled (VMT): Total amount of miles that all the vehicles have traveled over a certain section of freeway over a certain period of time.

Delay: Amount of additional time that vehicles spend on the roadway due to congestion.

Productivity Loss: Measure of the equivalent lane miles lost due to the freeway operating in congestion instead of at peak efficiency.

\section{CALIBRATION}

The calibration of the model comprises two main steps: 1 ) The calibration of the fundamental diagram for each link of the freeway, 2) Estimation of ramp flows, which are essential inputs to the simulation but are not monitored by PeMS and thus have to be imputed using the mainline flow data.

The first step of the fundamental diagram estimation is to plot the available PeMS data in a flow-density diagram. This scatter plot readily reveals that the typical shape of the fundamental diagram can be approximated by a triangle with piecewise linear free flow and congested regions separated by a certain critical density. Figure 3 shows the scatter plot and the fitted fundamental diagram for VDS 400443 on the studied freeway.

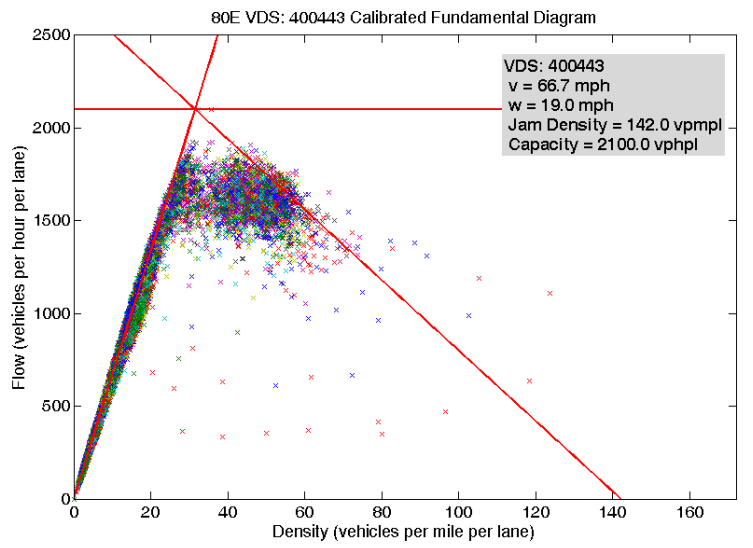

Fig. 3. Scatter Plot and fitted Fundamental Diagram

The estimation of the free flow parameter follows a simple linear regression whereas the congestion wave speed is estimated using an approximate quantile regression [6]. The capacity of the section is assigned as the maximum observed flow over the freeway segment. This VDS represents a typical cell for I-80 East with maximum flows around 2000 vehicles 
per hour per lane and a critical density around 35 vehicles per mile per lane. The estimated parameters for this particular section are stated in Figure 3.

The second part of the model calibration is the estimation of the missing ramp flows. Generally, data-based macroscopic freeway modeling is constrained by missing data. Despite the widespread collection of induction loop data in California, the simulation of I-80 suffers from the fact that no on- or off-ramp data is archived / readily available from the loop detectors present in the ramps. Therefore, an automated imputation procedure is implemented to estimate these values [7]. The imputation of unknown data uses adaptive identification techniques which are adopted from iterative learning control. The ramp flows and split ratios are estimated in two steps: In the first step, the input demands $c_{i}(k)$ for all links are simultaneously estimated using an iterative learning scheme. This identification scheme is model based, where the estimated parameters are used in the simulation and the error between the model calculated densities and measured densities are used to improve the parameter estimates. The LN-CTM simulation is performed several times and the total demands $c_{i}(k)$ are adjusted iteratively to minimize the density error of the simulation at each link in comparison with the real data. The simulation and parameters updates are repeated multiple times, so that the overall density error is minimized. The iteration for the density profile is done multiple times, always using the parameters from the previous run, so that the overall density error is minimized. The density error is the sum of the differences between the imputed and the measured densities $\Sigma_{(i, k)}\left|n_{i}(k)-\hat{n}_{i}(k)\right|$, where $\hat{n}_{i}(k)$ is the model calculated density estimate. The algorithm is terminated once the error reaches negligible values or stops decreasing across multiple runs.

In the next step, the on-ramp demands and the off-ramp split ratios are determined by solving a linear program. The input demands from the first step can be used to specify the input and output flows from links, and the flow measurements are available right in between the offramps and onramps on the freeway (Figure 4). Thus, it is possible to calculate the ramp flows by minimizing the error between the model calculated flows and the flow measurement between the ramps.

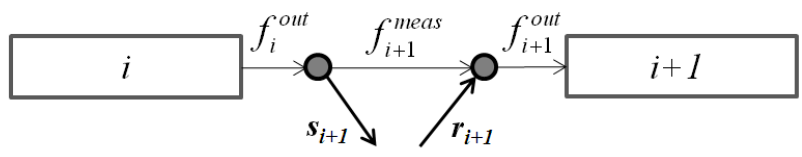

Fig. 4. Actual position of ramps and detector at a junction

One point of concern in the imputation process is the low quality of measurement at certain mainline vehicle detector stations and their diagnosis. A set of ad-hoc detection and correction measures were taken to discard incorrect data from the imputation procedure. The main approach to identify irregularities was a systematical analysis of the imputation results. Once the vehicle detector stations that report suspicious data are flagged, the imputation is run again, this time omitting the data from flagged detectors, and the results improve in terms of total error in density and flows. Overriding a flagged VDS results in the reduction of the freeway model since the cell it belongs to is now attached to the preceding cell upstream as shown in Figure 5 and the freeway model now consists of one less cell whereas the ramps of the adjoined cells are bundled together to represent a single on- or off-ramp each.

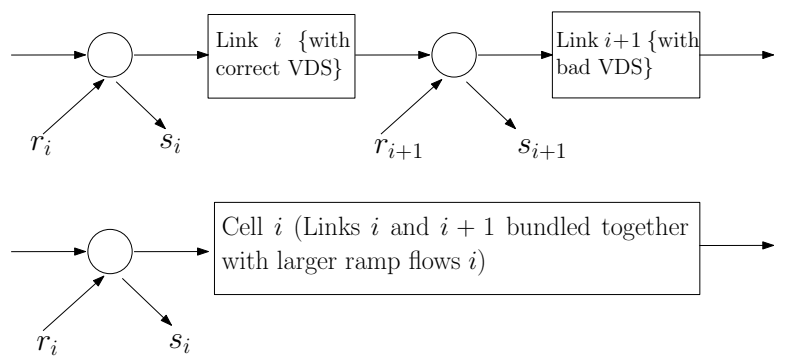

Fig. 5. Link structure before and after overriding link $i+1$

A graphical comparison procedure was used to flag the faulty detectors. For each VDS, the measured data of density and flow are compared to the simulated data, which is based on the imputation. In addition, it is useful to review the estimated on- and off-ramp flows as well as the presence or absence of on- or off-ramps. Thus, a graphical overview of the crucial factors is established as seen in Figure 6.
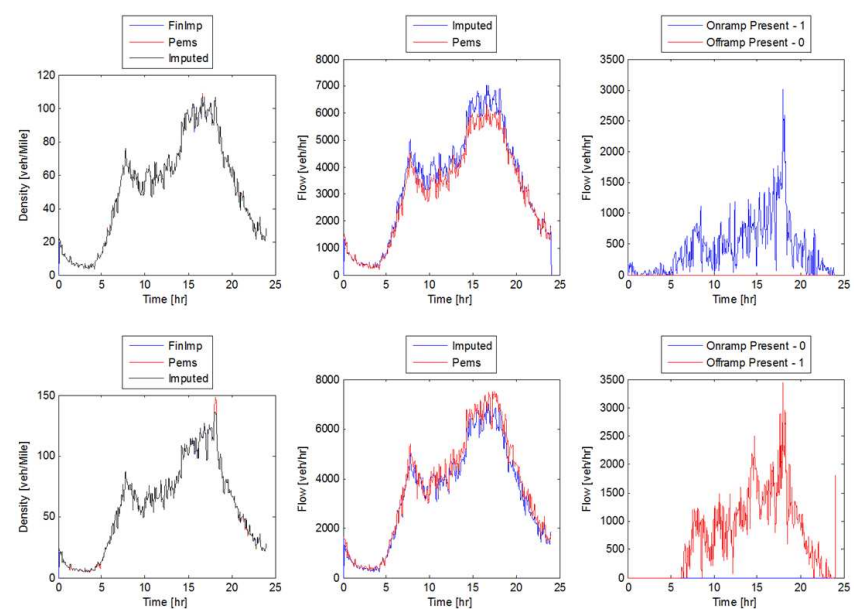

Fig. 6. Density, flow and ramp-flow plots for VDS 400976 (top) and 400838 (bottom)

The plots show an example of two VDSs which show almost perfect convergence between the imputed and the PeMS data for both density (left) and flow (middle) plots. It also gives the information that the cell of VDS 400976 (top) possesses just an on-ramp ("Onramp Present - 1"; "Offramp Present - 0") and the cell of VDS 400838 (bottom) just an off-ramp. The on-ramp flow is plotted in blue and the offramp flow is plotted in red (right plots). Reviewing these plots consecutively, it is possible to examine the longitudinal 
development of the daily flow and density characteristics. This makes it possible to see any disagreement between the simulated and measured densities and flows. A distinction of cases can be made in this analysis. The procedure begins with an analysis of observed density errors, then an analysis of flow errors is performed and, finally, faulty detectors are identified by density/flow mismatch.

Density errors in a link can be produced by faulty detectors in the upstream and/or downstream links, and depend on the prevalent mode of the LN-CTM (see figure 7).

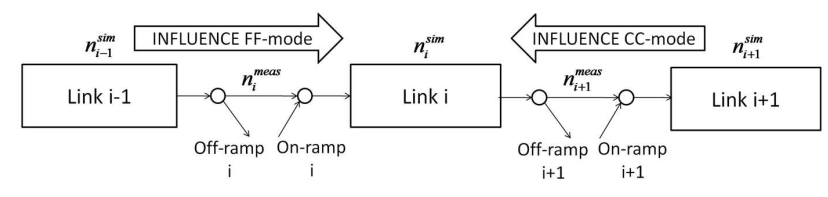

Fig. 7. Mode-dependent Influence on simulated density

For instance in the FF mode, the simulated density of link $i$ can only be influenced from upstream, i.e. increased over onramp $i$ and decreased over off-ramp $i$. Vehicles downstream cannot be queued because the traffic is in free-flow and therefore the ramp flows over on-ramp $i+1$ and off-ramp $i+1$ cannot influence the density of link $i$. Therefore, in the FF mode, a discrepancy in the measured and simulated densities of link $i$ can only be attributed to the ramp-flow estimation for ramps that precede link $i$. If, for instance, onramp $i$ does not exist and $n_{i}^{\text {sim }}$ is lower than $n_{i}^{\text {meas }}$, while $n_{i+1}^{\text {sim }}$ has converged to $n_{i+1}$, it means that the measured density of link $i-1, n_{i-1}^{\text {meas }}$, is suspiciously low (or $n_{i}^{\text {meas }}$ is suspiciously high) since no on-ramp exists to compensate for the missing vehicles to match $n_{i}^{\text {meas }}$ in the simulation.

In case of the CC-mode, the simulated density of link $i$ is influenced by the downstream ramp flows. This is due to the fact that the total flow entering link $i+1$ (i.e. the sum of the on-ramp flow and the flow from link $i$ into link $i+1)$ equals its capacity. Thus, the total flow leaving link $i$ is influenced by both on-ramp $i+1$ and off-ramp $i+1$. Hence, significantly low simulated densities $n_{i}^{\text {sim }}$, as compared to measurements $n_{i}^{\text {meas }}$ (when $n_{i+1}^{\text {sim }}$ has converged to $n_{i+1}^{\text {meas }}$ ), can be explained by one of the following: (1) $n_{i+1}^{\text {meas }}$ is too low (or $n_{i}^{\text {meas }}$ too high) and there is no on-ramp in between which can increase $n_{i}^{\text {sim }}$. (2) The fundamental diagram parameters of link $i$ have low estimated values because of faulty measurements, so that the output capacity of link $i-1$ is low. (3) The fundamental diagram parameters of link $i+1$ have high estimated values because of faulty measurements, so that the output capacity of link $i$ is high. Similarly, significantly high simulated densities $n_{i}^{\text {sim }}$ can be explained by one of the following: (1) $n_{i+1}^{\text {meas }}$ is too high (or $n_{i}^{\text {meas }}$ too low) and there is no off-ramp in between which could decrease $n_{i}^{\text {sim }}$. (2) The fundamental diagram parameters of link $i$ have high estimated values because of faulty data, so that the output capacity of link $i-1$ is high. (3) The fundamental diagram parameters of link $i+1$ have low estimated values because of faulty measurements, so that the output capacity of link $i$ is low.

Fault detection using flow data is comparitively simple.
For example, if there is no ramp between two consecutive cells and the flow plots differ, it is very likely that one VDS is reporting wrong values, since vehicle conservation dictates that the passing vehicle count per time period should not vary considerably. Similary, if there exists no onramps (offramps) in-between but the relative flow increases (decreases) significantly over succesive flow measurement stations, one of the flow measurements is faulty.

It is also possible that simulated and measured values converge smoothly in spite of the fact that the data is corrupted. For example, in the case where both on-ramp and off-ramp are present between cells, the occurrence of high imputed onramp flows upstream of the cell and high imputed off-ramp flows downstream is a good indicator of this phenomenon. If, in addition, those ramp-flows show unlikely profiles, the mainline data may be faulty and the large amounts of imputed ramp-flows only imitate the incorrectness of the measured data.

The described methods to find out incorrect measurements often narrow the choice of the bad detector to a few rather than pin-pointing the exact malfunctioning detector. To distinguish between the good and the bad detectors, it is useful to consider the plausibility of the candidate detectors' plots. There are three indicators which help to identify the bad VDS.

Midnight values: For both the density as well as the flow plots, the boundaries, i.e. the hours around midnight, provide good evidence whether the measurements are correct. If the densities and flows reported at night are unreasonably high or low, this VDS is likely to be the bad one.

Maximum values: Another aspect that indicates the bad detector can be found in the maximum values of the plots. If they seem too high or too low compared to the surrounding cells and the whole freeway, the VDS might be faulty.

Exceptional aspects: In addition to the two indicators above, the overall shape of the plots should be examined for exceptional aspects, such as the overall shape, which may indicate the existence of a VDS that reports false data.

Speeds: If the speeds across a section of the freeway are significantly low, it is an indicator for a faulty detector at this section.

If none of the fault detection techniques described above provide a reliable indication of which detector is faulty and instead point to several possible fault scenarios, several imputation/simulation trials must be performed to explore all possible fault scenarios and the scenario which results in the best overall imputation result is chosen.

\section{SIMULATION RESULTS}

For the simulation, the whole freeway segment is divided into a certain number of cells. This is performed by assuming one link for every VDS except for the case when there is a change in the number of lanes within the link, which results in a partition of the link into several links according to the segments with constant number of lanes. All links which belong to one VDS form one cell. To clarify the denotation: Each VDS represents one cell, but one cell can be partitioned 
into several links. The next step is to determine the locations of the ramps and assign them to their corresponding nodes in the freeway geometry. Once the geometric modeling is done, the calibration and ramp-flow imputations are carried through as described in the previous section and the freeway is ready to be simulated for the given demand and parameters. The following figures summarize the results of the simulation and compare them to the corresponding observations.
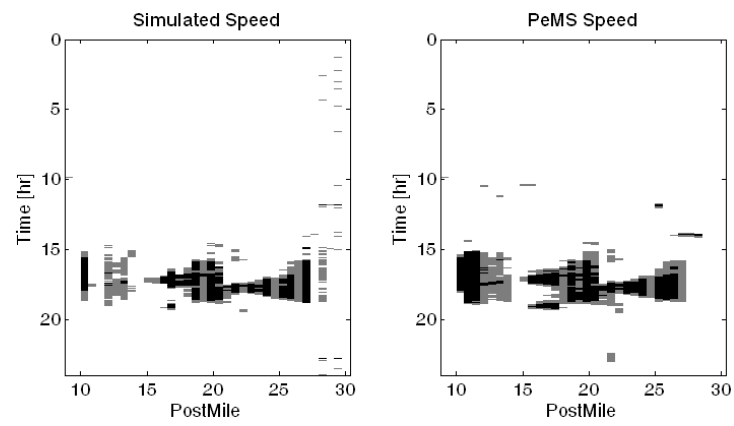

Fig. 8. Simulated vs Measured Density contours of I-80 E
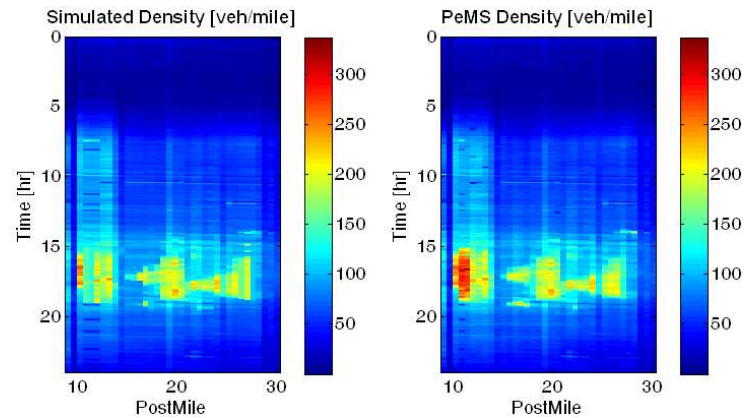

Fig. 9. Simulated vs Measured Speed Contours of I-80 E

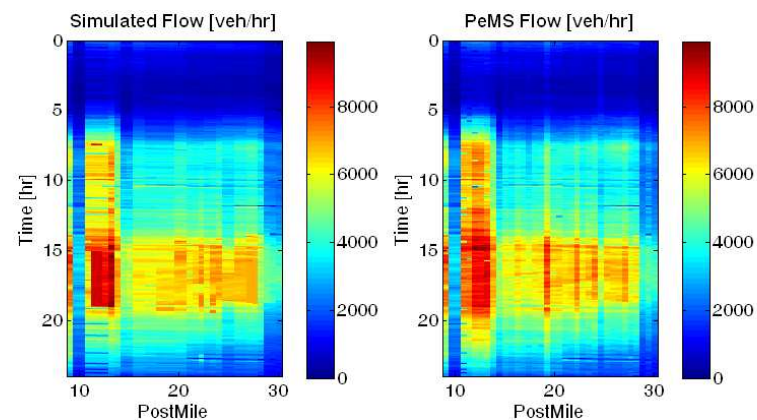

Fig. 10. Simulated vs Measured Flow Contours of I-80 E

Figures 8, 9 and 10 reflect the densities, speeds and flows across the freeway, respectively. On the horizontal axis are the successive links of the freeway in the order they appear in the direction of traffic flow and on the vertical axis is the time of the day for which the freeway was calibrated and simulated. Figure 11 reflects the measured vs simulated vehicle miles traveled and vehicle hours traveled. In addition to the visually clear agreement of the figures, the numerical values of the total errors for these three measures are quite satisfactory. The total density error was calculated to be $3.3 \%$, whereas the total flow error amounted to $7.1 \%$, which were decreased from $9.0075 \%$ and $15.4767 \%$, respectively, after the fault detection was carried out.
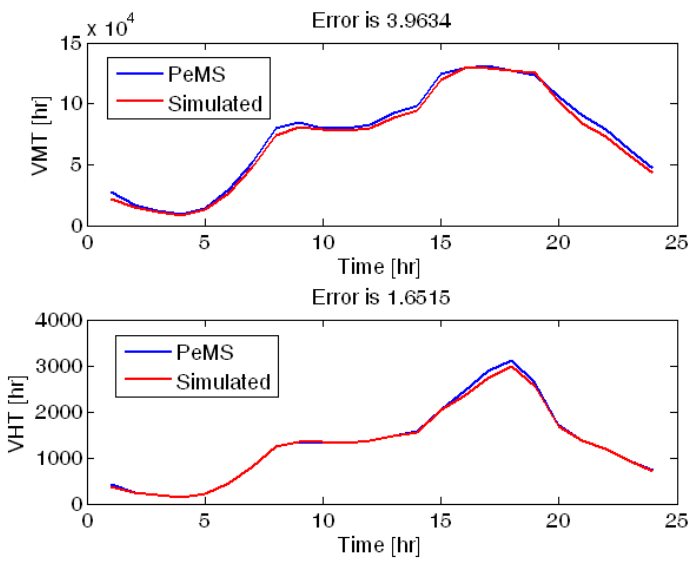

Fig. 11. Vehicle Miles Traveled and Vehicle Hours Traveled on I-80 E

\section{CONCLUSION}

The modeling and calibration of I-80E based on the LNCTM model has been elaborated in this paper. Overall, the macroscopic modeling of I-80E has proven the big service capability of macroscopic traffic models. Two main difficulties had to be overcome in the procedure: 1) Missing ramp data had to be estimated for the whole modeled freeway section, which has been achieved using an automated imputation procedure, 2) Huge extents of false measurements had to be identified and discarded using a graphical comparative data analysis. The results represent a functional calibrated model of I-80 East and can be used for further treatment, such as the implementation of different control strategies. The simulations, using the calibrated fundamental diagram data as well as the imputed on-ramp flows and off-ramp split ratios, agree closely with the measurements, as shown by the contour plots and performance curves presented in the previous section.

REFERENCES

[1] C. Daganzo, "The cell transmission model: A dynamic representation of highway traffic consistent with the hydrodynamic theory," Transportation Research, Part B, vol. 28, no. 4, pp. 269-287, 1994.

[2] G. Gomes and R. Horowitz, "Optimal freeway ramp metering using the asymmetric cell transmission model," Transportation Research, Part C, vol. 14 , no. 4, pp. 244-262, 2006.

[3] A. Kurzhanskiy, Modeling and Software Tools for Freeway Operational Planning. PhD thesis, University of California, Berkeley, 2007.

[4] PeMS, "PeMS website," 2007. http://pems.eecs.berkeley.edu, accessed 8/28/2007.

[5] A. Muralidharan, G. Dervisoglu, and R. Horowitz, "Freeway traffic flow simulation using the cell transmission model," American Controls Conference, 2009.

[6] G. Dervisoglu, G. Gomes, J. Kwon, R. Horowitz, and P. Varaiya, "Automatic calibration of the fundamental diagram and empirical observations on capacity," Transportation Research Board, 88th Annual Meeting, 2009.

[7] A. Muralidharan and R. Horowitz, "Imputation of ramp flow data for freeway traffic simulation," Transportation Research Board, 88th Annual Meeting, 2009. 